ITFA-93-39

November 1993

\title{
Some non-perturbative aspects of gauge fixing in two-dimensional Yang-Mills theory
}

\author{
James E. Hetrick ${ }^{\mathrm{a}}$

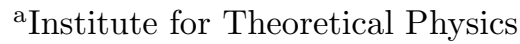 \\ University of Amsterdam \\ Valckenierstraat 65 \\ 1108-DX Amsterdam, The Netherlands
}

\begin{abstract}
Gauge fixing in general is incomplete, such that one solves some of the gauge constraints (fixes to some gauge), quantizes, then imposes any residual gauge symmetries (Gribov copies) on the wavefunctions. While the FadeevPopov determinant keeps track of the local metric on this gauge fixed surface, the global topology of the reduced configuration space can be different depending on the treatment of the residual symmetries, which can in turn affect global properties of the theory such as the vacuum wavefunction.

Pure $S U(N)$ gauge theory in two dimensions provides a simple yet non-trivial example where the above structure and effects can be elucidated explicitly, thus displaying physical effects of the treatment of Gribov copies.
\end{abstract}

\section{Introduction}

The essential nature of a gauge theory is of course its gauge symmetry, whereby a fiber円 of gauge transformed fields desribes a unique physical configuration. Symbolically we represent this structure with the following icon

$$
\begin{array}{cl}
\mathcal{G} \longrightarrow \quad & \mathcal{Q} \\
& \downarrow \Pi \\
& Q_{\text {physical }}
\end{array}
$$

where the base space of physical configurations $Q_{\text {physical would have coordinates } z \text {, while the fiber }}$ bundle $\mathcal{Q}$ has coordinates $A_{\mu}(x)=(z, \Omega)$, with $\Omega$ parametrizing the fiber. The meaning of $\mathcal{G} \rightarrow \mathcal{Q}$ is that the group $\mathcal{G}$, the set of all possible gauge transformations, acts on the fiber coordinates $(z, \Omega) \rightarrow\left(z, \Omega^{\prime}\right)$. The projection $\Pi$ projects from $\mathcal{Q}$ down to $Q_{\text {physical }} \Pi:(z, \Omega) \rightarrow z$.

Gauge fixing the theory, means finding a unique point up in the bundle for each physical configuration $z \in Q_{\text {physical }}$, ie. an inverse projection $\Pi^{-1}: z \rightarrow(z, \Omega(z))$, which is called a section of the bundle. Classically, variation of the La-

\footnotetext{
${ }^{1}$ I will use freely the language of fiber bundles, which is the natural mathematical setting of gauge theories. For an excellent introduction to the geometry and topology of fiber bundles, see [1]
}

grangian gives the equations of motion of a trajectory $z(t)$ on the base space $Q_{\text {physical }}$ and any lift of this trajectory to a section in the bundle will satisfy the equations of motion. The choice of this section or gauge, is a matter of computational convenience for the problem at hand.

In a quantum theory of gauge fields the situation is greatly changed, since the wave functions, or the measure in the path integral, are sensitive to the global geometry of the configuration space, rather than just the local structure of phase space as in the Hamiltonian evolution of the classical system. This allows for some ambiguity in the definition of the quantum gauge theory, since we have the choice of either solving the constraints first, then quantizing on the reduced configuration space, or quantizing first, and implimenting the gauge symmetries as constraints (symmetries) of the wave functions.

Usually we proceed with some combination of the above for technical reasons, whereby we gauge fix the fields to some convenient gauge, which leaves residual gauge symmetries still acting on the reduced configuration space. These we impliment as symmetries on the wave functions. Such are the residual homotopically non-trivial gauge transformations which generate the $\theta$ vacua, as 
well as residual Gribov copies which occur in nonAbelian gauge theories. Scematically we can represent this structure with the following diagram:

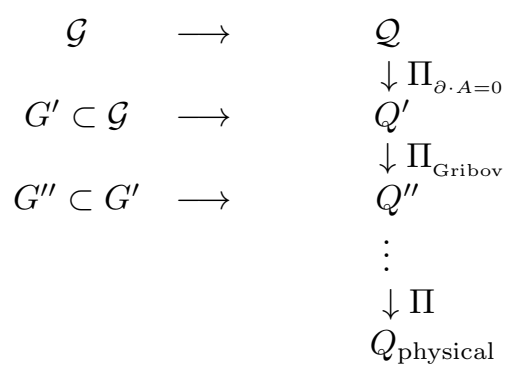

The local geometry (metric) of the successive configuration spaces $\mathcal{Q}, Q^{\prime}, Q^{\prime \prime}, \ldots$ is taken care of by the Fadeev-Popov determinant as the Jacobian of the projection down to the reduced configuration space at each stage. This insures that the perturbative structure is intact for any gauge.

The successive residual symmetries contain detailed information about the global topology of the fundamental modular domain, $Q_{\text {physical }}$, which is a crucial aspect of the non-perturbative structure of the theory. As the lattice provides the only non-perturbative means of calculation at present, it is important to understand the emergence of the topology of the configuration space, the treatment of the Gribov copies, and the artifacts in the gauge orbit space due to the lattice.

The quantum theories defined at different levels $Q^{\prime}, Q^{\prime \prime}, \ldots$ are defined on configuration spaces of different topology, since at each level more of the residual constraints have been solved and the configuration space compactified accordingly. These different topologies of $Q^{\prime}$ and $Q^{\prime \prime}$ will affect the types of Hilbert spaces which can be constructed over them. For instance, a general wave function on a circle $S^{1}$ can have a phase $e^{i \alpha}$ upon transport around the circle. Although this circle is a submanifold of an $n$-sphere, and hence obtained by some projection $\Pi_{S^{n} \rightarrow S^{1}}$, wave functions on $S^{n}$ must be single valued since $\pi_{1}\left(S^{n}\right)=0$ for $n>1$. In general we may find that the topologies of $Q^{\prime}$ and $Q^{\prime \prime}$ dictate incomensurate Hilbert spaces, in which case we have inequivalent quantizations of the gauge theory. Such is the case for $S U(N)$ gauge fields in two spacetime dimensions 近幽.

\section{2. $S U(N)$ gauge theory in two dimensions}

We consider periodic $S U(N)$ gauge fields $A_{\mu}(x)$ on a cylindrical spacetime $(x, t) \in S^{1} \times R^{1}$ using canonical quantization to illuminate the different Hilbert spaces that emerge depending on the level of gauge fixing; details can be found in [2].

First we identify the fundamental modular domain $Q_{\text {physical }}$ explicitly, which is possible due to the simple structure of a gauge theory in two dimensions. As explained in [2], the set of physically distinct field configurations is isomorphic to the space of conjugacy classes of $G=S U(N)$. This space is an orbifold, which we label $\Xi_{G}$, made by identifying the points in the maximal torus of $G$ (the Cartan subgroup) under reflections in the diagonal planes of the torus: $\theta_{i} \leftrightarrow \theta_{j}$. Since these reflections invert the tangent plane, $\Xi_{G}$ will be non-orientable, ie. it has no globally defined tangent bundle. This is an essential feature of the global topology of the fundamental modular domain and will affect the types of wave functions it admits.

For a concrete example consider $G=S U(2)$. In fact we can almost fix the gauge completely to $Q_{\text {physical }}$ if $A_{1}$ satisfies the Coulomb gauge condition, $\partial_{1} A_{1}=0$, and is further diagonal. Then

$A_{1}(x, t)=\left(\begin{array}{ll}\theta(t) & \\ & -\theta(t)\end{array}\right)$

corresponding to a configuration space $Q^{\prime \prime}$ just above the fundamental moduli space $Q_{\text {physical }}$ with only discrete residual symmetries left, namely: $\theta \rightarrow \theta+n \pi$ and $\theta \rightarrow-\theta$. It is the latter transformation which makes the circle defined by $\theta$ into the non-orientable orbifold $\Xi=(0, \pi)_{ \pm}$. By $(0, \pi)_{ \pm}$is meant that $\Xi_{S U(2)}$ is described by two patches $(0, \pi)$ which cover the same points but have oppositely oriented tangent spaces.

In this gauge we easily find that the Hamiltonian is simply the Laplacian on the circle

$H=-\frac{g^{2} L}{8} \frac{\partial^{2}}{\partial \theta^{2}}$

where $g$ is the coupling constant and $L$ is the volume of space. In general the wave function $\Psi_{n}(\theta)$ could be some combination of $\exp [ \pm i 2 \pi \alpha(\theta+\beta)]$, however non-integer $\alpha$ implies some some physics 
(Bohm-Aharonov phenomena) at $\theta=0$ or $\pi$. That $\Xi_{S U(2)}$ is non-orientable in fact determines $\Psi_{n} \sim \cos (n \theta)$ since it is this wave function whose momentum vanishes at the orbifold singularities where the tangent space is ill defined [5, 6]. The spectrum in this case is

$E_{j}=j^{2}$

and $j=0, \frac{1}{2}, 1, \frac{3}{2}, \ldots$

If on the other hand we gauge fix only to the Coulomb gauge condition, $\partial_{1} A_{1}=0$, the configuration space is isomorphic to the group manifold $S U(2)=S^{3}$ itself, and the residual gauge symmetries are all constant adjoint rotations of $A_{1}$. Then the Hamiltonian is the Laplacian on $S U(2)$ and wave functions are characters $\chi_{j}(\theta)$, which are invariant under adjoint action of $G$ on itself. In comparing the spectrum on $G$ to the one above (on the flat maximal torus), there is a constant shift in energy proportional to the curvature of $G$. This then yields the spectrum

$E_{j}=j(j+1)+\frac{1}{4}=\left(j+\frac{1}{2}\right)^{2}$

with $j$ taking half integer values as above. The two spectra agree except for the vacuum states as depicted in figure 1 .

The difference is that quantizing on the maximal torus, preserving the non-orientable nature of $Q_{\text {physical }}$ chooses even (cos) fourier modes on the maximal torus, However only the odd ( $\sin )$ modes have a globally defined lift to the gauge group manifold. Characters

$\chi_{j}=\frac{\sin [(j+1 / 2) \theta]}{\sin (\theta / 2)}$

are odd fourier modes, $\sin [(j+1 / 2) \theta]$, divided by the square root of the Haar measure (also odd). This construction is general and the shift of $j$ by $1 / 2$ is so that $\chi_{j=0}=1$. The lift of the even $(\cos )$ mode: $\cos (j \theta) / \sin (\theta / 2)$ is in fact an eigenfunction of the Laplacian with eigenvalue $j(j+1)$, and while square integrable, is not a single valued function on $S U(2)$.

The lattice, essentially due to it's compact formulation on $G$ from the start, gives the latter quantization above, without gauge fixing. It will

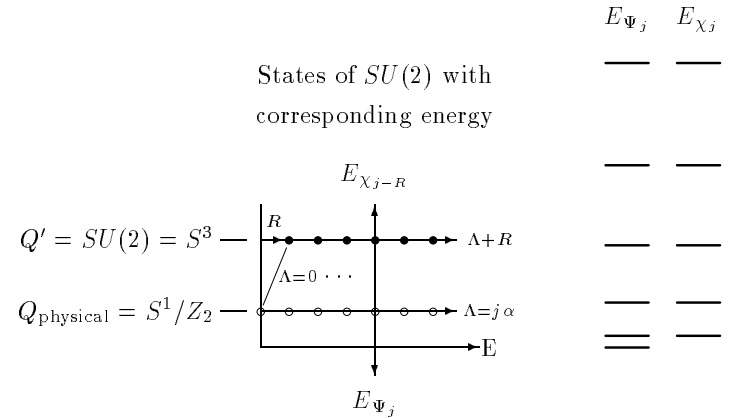

Figure 1. Correspondence of the excited spectra and the shift of ground state for two different gauge choices. States are labeled by irreducible representations $\Lambda=j \cdot \alpha$ of $S U(2)$, where $\alpha$ is the normalized root vector. $R$ is the shift due to the curvature of $G$ and equals half the sum of the positive roots. See [2] for further details.

be very interesting to see what type of gauge fixing is necessary in the lattice theory to reproduce the former spectrum, and if this can indeed be done in the compact theory. Such a program is underway.

This work is supported by the Stichting voor Fundamenteel Onderzoek (FOM).

\section{REFERENCES}

1. Geometry, Topology, and Physics, M. Nakahara, Hilger (1990); Bristol, UK (Graduate student series in physics)

2. J.E. Hetrick "Canonical quantization of two dimensional gauge fields", hep-lat/9305020. to appear in Int. J. Mod. Phys. A

3. J. Hallin, Gothenburg ITP-93-33, "SU $(N)$ Yang-Mills on a circle, loop variables, Mandelstam identities and quantization ambiguities", hep-th/9308015

4. L. Chandar and E. Ercolessi, SU-4240-537, Inequivalent quantizations of Yang-Mills theory on a cylinder, hep-th/9309065

5. E. Farhi and S. Gutmann, Int. J. Mod. Phys. A5, (1990) 3029

6. J.S. Dowker, J. Phys. A10 (1977) 115 